\title{
REGISTRO DE DADOS SOBRE O USO DE IMUNOBIOLÓGICOS E INSUMOS NAS SALAS DE VACINAS*
}

\author{
Taís Trombetta Dalla Nora ${ }^{1}$, Giulliana Weigert Herrera², Graciele Fernanda da Costa Linch ${ }^{3}$, Adriana \\ Aparecida $\mathrm{Paz}^{3}$
}

RESUMO: Objetivo: verificar os registros dos dados sobre o uso de imunobiológicos e insumos nas salas de vacinas da rede pública. Método: estudo transversal, realizado em oito salas de vacinas da atenção básica, com 106 observações em novembro e dezembro de 2016, em um município do Rio Grande do Sul. Resultados: em $64(60,4 \%)$ atendimentos os usuários estavam cadastrados no programa e 104(98,1\%) tiveram as vacinas registradas corretamente. Os dados foram registrados adequadamente após a vacinação do usuário no programa em $89(84 \%)$ atendimentos, entretanto, a movimentação do imunobiológico foi preenchida em 17(11\%) observações. O registro no boletim diário da temperatura do refrigerador ocorreu em 80(75,5\%) observações. Conclusão: foi possível obter subsídios para a elaboração de ações estratégicas no município, com a finalidade da qualificação do processo de trabalho quanto à organização, manutenção e registros dos imunobiológicos nas salas de vacina. DESCRITORES: Enfermagem; Programas de imunização; Sistema de registros; Registros de enfermagem; Atenção Primária à Saúde.

\section{REGISTRATION OF DATA ON THE USE OF IMMUNOBIOLOGICALS AND SUPPLIES IN VACCINE ROOMS}

ABSTRACT: Objective: To verify the registration of the data on the use of immunobiologicals and supplies in the vaccination rooms of the public network. Method: a cross-sectional study, carried out in eight primary care vaccination rooms, with 106 observations in November and December 2016, in a municipality of Rio Grande do Sul. Results: in $64(60.4 \%)$ consultations the clients were enrolled in the program and $104(98.1 \%)$ had their vaccinations registered correctly. Data were adequately recorded after the vaccination of the user in the program in $89(84 \%)$ consultations, however, the movement of the immunobiological agent was only completed in 17 $(11 \%)$ observations. The refrigerator temperature was registered in the daily record in $80(75.5 \%)$ observations. Conclusion: the study provided support for the elaboration of strategic actions in the municipality, with the aim of qualifying the work process regarding the organization, maintenance and registration of the immunobiologicals in the vaccine rooms.

DESCRIPTORS: Nursing; Immunization programs; System of records; Nursing records; Primary Healthcare.

\section{REGISTRO DE DATOS ACERCA DE USO DE INMUNO BIOLÓGICOS E INSUMOS EN LOS LOCALES DE VACUNACIÓN}

RESUMEN: Objetivo: verificar los registros de los datos acerca del uso de inmuno biológicos e insumos en las salas de vacunación de la red pública. Método: estudio transversal, que se realizó en ocho salas de vacunación de la atención básica, con 106 observaciones en noviembre y diciembre de 2016, en un municipio de Rio Grande do Sul. Resultados: en 64(60,4\%) atendimientos, los usuarios estaban registrados en el programa y $104(98,1 \%)$ tuvieron las vacunas registradas correctamente. Se registraron los datos de modo adecuado tras la vacunación del usuario en el programa en $89(84 \%)$ atendimientos. Sin embargo, se completó el movimiento del inmuno biológico en $17(11 \%)$ observaciones. El registro en el boletín diario de la temperatura del refrigerador ocurrió en $80(75,5 \%)$ observaciones. Conclusión: fue posible obtener subsidios para la elaboración de acciones estratégicas en el municipio, con la finalidad de calificar el proceso de trabajo en cuanto a la organización, manutención y registros de los inmuno biológicos en las salas de vacunación.

DESCRIPTORES: Enfermería; Programas de inmunización; Sistema de registros; Registros de enfermería; Atención Básica a la Salud.

*Artigo extraído da dissertação de mestrado "Intervenção educativa na sala de vacinas: registros de imunobiológicos". Universidade Federal das Ciências da Saúde de Porto Alegre, 2017.

${ }^{1}$ Enfermeira. Mestre em Enfermagem. Especialista em Saúde da Secretaria Estadual de Saúde do Rio Grande do Sul, lotação 19a CRS. Frederico Westphalen, RS, Brasil.

${ }^{2}$ Discente de Enfermagem. Universidade Federal das Ciências da Saúde de Porto Alegre. Porto Alegre, RS, Brasil.

${ }^{3}$ Enfermeira. Doutora em Enfermagem. Docente de Enfermagem e de Pós-Graduação em Enfermagem da Universidade Federal das Ciências da Saúde de Porto Alegre. Porto Alegre, RS, Brasil.

Autor Correspondente:

Taís Trombetta Dalla Nora

$19^{a}$ Coordenadoria Regional de Saúde

R. Monsenhor Vitor Batistela, 576 - 98400-000 - Frederico Westphalen, RS, Brasil

E-mail: taistrombetta@yahoo.com.br
Recebido: 10/11/2017

Finalizado: $22 / 08 / 2018$ 


\section{- INTRODUÇÃO}

A vacinação é ação estratégica e integrada dos serviços de saúde, disponível na Atenção Básica ou nos serviços de saúde suplementar. Agregada às ações de prevenção e de proteção, a vacina potencializa as medidas de bloqueio de doenças imunopreveníveis. Os avanços da tecnologia em imunobiológicos e saúde nas últimas décadas constituem-se como estratégia de melhor relação custo e efetividade no setor saúde ${ }^{(1)}$.

O Programa Nacional de Imunização (PNI) brasileiro é reconhecido por diversos países como um sistema complexo que garante e dispõe os imunobiológicos à população nas instâncias dos serviços do Sistema Único de Saúde (SUS). A gestão do PNI promove avanços na medida em que se consolida pelo incremento de novas vacinas, ampliação do acesso de populações vulneráveis, aumento da cobertura vacinal, bem como eliminação e prevenção de doenças imunopreveníveis ${ }^{(2)}$.

No PNI, o enfermeiro tem a responsabilidade de organizar as atividades da equipe e participar de ações como intensificação, bloqueio e campanhas de vacinação. No cenário assistencial da atenção básica, o enfermeiro realiza e/ou supervisiona o técnico de enfermagem na atualização do cartão vacinal do usuário, na administração de vacinas, nas solicitações de insumos para as salas de vacinas, e na orientação dos usuários e equipe de saúde sobre as principais ações para o bloqueio e controle de doenças imunopreveníveis ${ }^{(3)}$.

Diante dessas atribuições, o enfermeiro precisa desenvolver na sua prática gerencial a proposição de ações educativas e o monitoramento de salas de vacina, para o alcance de resultados definidos no planejamento relacionado às coberturas vacinais ${ }^{(4)}$.

Para o registro informatizado dos dados de vacinação, no Brasil, é utilizado o Sistema de Informação do PNI (SIPNI). O programa é desenvolvido para uso em Desktop na modalidade off-line, tendo as atualizações periódicas pela Internet. Esse sistema de informação registra nominalmente os vacinados a partir do cadastro do Cartão SUS e os imunobiológicos administrados ${ }^{(5)}$.

Além disso, o programa gera dados da movimentação de imunobiológicos nas salas de vacinas, sendo este o único meio de transmissão de dados para o $\mathrm{PNI}^{(6)}$. Além desse sistema, utiliza-se o Sistema de Informação de Insumos Estratégicos (SIES) para a solicitação de imunobiológicos e insumos diversos. Trata-se de um sistema online que necessita de login e de senha do usuário, e por meio do qual também se controla o estoque das salas de vacinas pela gestão municipal, estadual e nacional(5).

Até meados de 2012, os registros eram realizados manualmente em planilhas que eram agrupadas, e ao final do mês fazia-se um relatório eletrônico com os dados sobre o uso de imunobiológicos e insumos às gestões do PNI. A partir de 2013, os municípios passaram a realizar os registros de dados de imunobiológicos e insumos em tempo real, o que possibilitou ampliar as estratégias de gestão relacionadas às doenças imunopreveníveis ${ }^{(6)}$.

Entretanto, no município cenário do estudo, observou-se um decréscimo nos indicadores da cobertura vacinal com o passar dos anos, mesmo com a plena cobertura de equipes de saúde da família em todo o território, a realização de buscas ativas pelos agentes comunitários de saúde e as campanhas nacionais e locais.

Assim, diante desse contexto instalado e vivenciado na atenção básica municipal e sendo uma das pesquisadoras a enfermeira Coordenadora Municipal do PNI, surgiu a questão de pesquisa: "Como ocorrem os registros de dados sobre o uso de imunobiológicos e insumos nas salas de vacinas da rede pública?". Desta maneira, este estudo teve o objetivo de verificar os registros dos dados sobre o uso de imunobiológicos e insumos nas salas de vacinas da rede pública.

\section{MÉTODO}

Trata-se de uma pesquisa com delineamento transversal ${ }^{(7)}$. O estudo foi realizado nas oito salas de vacinas da Atenção Básica em um município de pequeno porte, localizado na Região Noroeste do Rio Grande do Sul. Nesse município, a Atenção Básica abrange $100 \%$ do território, havendo oito unidades 
de saúde com Estratégias de Saúde da Família que atendem a população urbana e rural.

Foram observados 106 registros dos dados de imunobiológicos e insumos realizados por 15 profissionais de enfermagem. As observações abrangeram todos os profissionais envolvidos com atividades nas salas de vacinas. Salienta-se que nenhum profissional recusou participação no estudo, a qual se deu por meio da observação do exercício profissional dos participantes.

O critério de inclusão para se estabelecer a observação sobre os registros foi a realização do registro pelo profissional de enfermagem atuante em sala de vacinas da unidade de saúde do município. O critério de exclusão foi a inexistência de profissional de enfermagem na sala de vacinas por motivo de licença saúde, maternidade ou férias.

Participaram sete enfermeiras e oito técnicas de enfermagem, todas do sexo feminino e com a

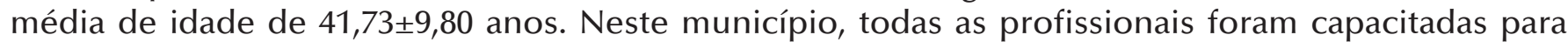
atuarem em sala de vacinas, além de exercerem outras atividades concomitantes de acordo com as demandas e as necessidades de atenção da população. O tempo de experiência em sala de vacinas das profissionais variou de 5 a 14 anos.

A coleta de dados ocorreu de novembro a dezembro de 2016, sendo utilizado um instrumento para a observação direta e sistemática não participante do processo de trabalho do registro de dados dos imunobiológicos e insumos. A observação sistemática utiliza instrumentos para a observação do fenômeno a fim de responder os propósitos preestabelecidos ${ }^{(8)}$.

A observação ocorreu em todas as oito salas de vacinas, em que a pesquisadora não se integrou ao processo de trabalho na resolução das situações, cabendo o papel de espectador da prática dos profissionais. Para dar início às observações, foram agendados previamente dois momentos em cada sala de vacina, em turnos opostos, com a equipe de enfermagem para a verificação dos registros de dados relacionados aos imunobiológicos e insumos.

Os dados das observações foram organizados e armazenados inicialmente em planilhas do Excel e posteriormente importados e analisados pelo programa Statistical Package Social Science (SPSS). A análise ocorreu pela estatística descritiva das variáveis categóricas que foram expressas em frequência absoluta e relativa. O estudo teve a aprovação do Comitê de Ética em Pesquisa da Universidade Federal de Ciências da Saúde de Porto Alegre, com parecer $\mathrm{n}^{\circ} 1.798 .843$ respeitando os preceitos éticos estabelecidos pela Resolução nº 466/2012 do Conselho Nacional de Saúde ${ }^{(9)}$.

\section{- RESULTADOS}

Foram realizadas 106 observações em oito salas de vacinação, com distribuição de 10 a 16 observações por sala. As observações dos registros ocorreram em maior número no turno da tarde $58(54,7 \%)$ devido à demanda dos usuários. Dentre os profissionais de enfermagem, a enfermeira realizou 59(55,7\%) registros de dados de imunobiológicos e insumos. Na Tabela 1 são apresentadas as observações realizadas sobre o registro de imunobiológicos e insumos.

Tabela 1 - Situações afirmativas nas observações realizadas nas salas de vacinas de um município do Noroeste do Rio Grande do Sul, RS, Brasil, 2017 (continua)

\begin{tabular}{lc} 
Variáveis & $\mathbf{n ( \% )}$ \\
\hline Registro prévio do usuário no SIPNI & $64(60,4)$ \\
\hline Disponibilidade de informativo do calendário do esquema vacinal & $94(88,7)$ \\
\hline Administração da vacina de acordo com a faixa etária do usuário & $54(50,9)$ \\
\hline Aprazamento das vacinas no cartão & $92(86,8)$ \\
\hline Orientação do retorno para vacinas aprazadas & $87(82,1)$ \\
\hline Adiamento para a aplicação de vacinas & $12(11,3)$ \\
\hline Registro no cartão de vacinas do usuário correto & $104(98,1)$ \\
\hline
\end{tabular}


Cogitare Enferm. (23)4: e56274, 2018

\begin{tabular}{lc}
\hline Registro dos dados no PNI correto & $89(84)$ \\
\hline Disponibilidade de vacinas de acordo com a demanda & $106(100)$ \\
\hline Registro da temperatura do refrigerador no boletim de controle diário & $80(75,5)$ \\
\hline Registro da temperatura dentro dos padrões & $86(81,1)$ \\
\hline Frascos multidoses rotulados & $82(77,4)$ \\
\hline Registro da movimentação de imunobiológicos & $17(16)$ \\
\hline Estoque atualizado no SIES & $59(55,7)$ \\
\hline Versão atualizada do SIPNI no Desktop & $61(57,5)$
\end{tabular}

Das observações realizadas, para usuários sem cadastro no SIPNI, o profissional de enfermagem realizou o cadastramento utilizando o número do cartão SUS. Foi verificado que em 94(88,7\%) observações, estavam disponíveis os informativos sobre o calendário de esquema vacinal, possibilitando o acesso rápido e fácil para esclarecimento de dúvidas e orientação dos usuários.

O esquema vacinal agrega o Calendário Nacional de Imunização que traz as indicações para a faixa etária do usuário para a administração da vacina. Foi identificado em 54(50,9\%) observações que os usuários estavam dentro da faixa etária exata recomendada. Constatou-se a realização do registro de aprazamento correto na carteira de vacinação em 92(86,8\%) observações, sendo informado o aprazamento das próximas vacinas. Em 87(82,1\%) observações, o profissional orientou verbalmente o usuário e acompanhante sobre a realização das próximas vacinas de acordo com o calendário de vacinas.

As Perdas de Oportunidades de Vacinação (POVs) são consideradas como adiamento da aplicação da vacina, sendo constatadas em $12(11,3 \%)$ observações. Os usuários tinham que receber múltiplas vacinas injetáveis no mesmo dia e, muitas vezes, por serem crianças, foi postergada a aplicação, de vacinas tais como os reforços da Pneumocócica 10, Meningocócica C e a primeira dose da Tríplice Viral (que no calendário de vacinas devem ser aplicadas quando a criança estiver com um ano de vida).

Durante a observação, em algumas salas de vacinas, essa indicação de adiamento foi sugerida pelo profissional de enfermagem, o que implica na reorganização do usuário para o retorno a unidade de saúde em outro dia; na suscetibilidade às doenças imunopreveníveis pela inexistência de bloqueio imunológico individual da criança; e no fato de que o profissional não efetuou a aplicação seguindo as orientações preconizadas pelo PNI.

Os registros de vacinas no cartão do usuário ocorreram corretamente em 104(98,1\%) observações, demonstrando a qualidade dessa etapa nos registros realizados pelos profissionais de enfermagem. Ainda, os registros de vacinas administradas no SIPNI, em 89(84\%) observações foram inseridos adequadamente, considerando o paciente certo, vacina certa, dose certa e lote certo. No que se refere à disponibilidade de vacinas, foi atendida a demanda em sua totalidade.

O registro de temperatura do refrigerador foi realizado no boletim de controle diário, com a marcação da temperatura do início e fim do expediente em $80(75,5 \%)$ observações. Essa planilha deve ser preenchida diariamente, nos dias úteis, quando ocorrem as atividades assistenciais da unidade de saúde. Na avaliação desses registros constatou-se que em $86(81,1 \%)$ observações a temperatura do refrigerador estava dentro do preconizado pelo PNI. Em relação aos frascos multidoses acondicionados nos refrigeradores, esses estavam e/ou foram rotulados em $82(77,4 \%)$ observações. A rotulagem dos frascos continha as informações do horário de abertura e de validade do frasco da vacina.

O registro de movimentação de imunobiológicos ocorreu apenas em 17(16\%) observações. Esses registros apresentam fragilidades que resultam do tempo de atendimentos dos usuários, da própria organização pessoal e laboral do profissional, das dificuldades com o uso da informática e do acesso ao SIPNI e SIES atualizados.

Quanto ao gerenciamento de estoque de vacinas e insumos no SIES, verificou-se que em 59(55,7\%) observações o profissional atualizou o estoque. Essa prática nem sempre ocorre imediatamente à administração da vacina, pois em algumas unidades de saúde a atualização é feita ao final do dia ou da semana. Em relação ao uso da versão atualizada do SIPNI no Desktop, identificou-se a ocorrência em 
$61(57,5 \%)$ observações. Essa atualização carece de atenção dos profissionais das salas de vacinas para que se tenha a última versão, pois, periodicamente, o PNI propõe diversas alterações para a melhoria do sistema.

\section{DISCUSSÃO}

O cadastramento do usuário no SIPNI possibilita avaliar com maior exatidão as características populacionais para as quais existe a necessidade de prover os imunobiológicos e insumos nas unidades de saúde. Na medida em que as salas de vacinas são informatizadas, esse cadastramento é exigido para que ocorra a vacinação do usuário em consonância ao calendário de vacinas. Desta maneira, o usuário cadastrado previamente permite verificar as vacinas administradas utilizando como identificador o número do cartão SUS. Nesta pesquisa, o cadastramento foi realizado corretamente em $98,1 \%$ das observações, por meio do cartão do SUS do usuário.

No estudo da cobertura vacinal, em Augusto Pestana-RS, foi observado que, para se ter um panorama real das atividades realizadas nas salas de vacinas utilizando o SIPNI, era necessário que 100\% dos usuários estivessem cadastrados no programa ${ }^{(10)}$. Portanto, o registro do usuário possibilita avaliar a situação da cobertura vacinal.

A disponibilidade de informativos contendo o calendário com o esquema vacinal ocorreu em $88,7 \%$ das observações neste estudo. O uso desses informativos serve como instrumento para auxiliar a assistência prestada em sala de vacina, esclarecendo dúvidas tanto aos profissionais como aos usuários. Geralmente, as coordenações municipais elaboram informativos utilizando os manuais do PNI publicados pelo Ministério da Saúde.

Em um estudo, foram observadas 41 salas de vacinas no município de Marília-SP, sendo identificado que em $39(95,1 \%)$ salas não havia o manual de capacitação de pessoal em sala de vacina disponível para consulta dos profissionais, e em $17(41,4 \%)$ salas não foram localizados o manual da rede de frio ${ }^{(11)}$. Em outro estudo com 15 profissionais atuantes em salas de vacinas, a maioria relatou a preocupação em explicar sobre as doenças imunopreveníveis, os eventos adversos e o manejo antes da aplicação da vacina ${ }^{(12)}$.

Em Recife-PE, estudo avaliou 300 cadernetas de saúde da criança (CSC), identificando que $40 \%$ estavam em atraso vacinal em comparação à idade recomendada pelo $\mathrm{PNI}^{(13)}$. No mesmo estudo, observou-se que todos os profissionais verificaram a idade e os intervalos entre as doses recomendados antes da administração das vacinas ${ }^{(13)}$. Neste estudo observou-se que $50,9 \%$ dos usuários que receberam as vacinas tinham a faixa etária recomendada para a vacina, não caracterizando o atraso vacinal.

O PNI preconiza que, ao utilizar vacinas, soros e imunoglobulinas, é necessário respeitar aspectos específicos, entre eles a faixa etária recomendada para cada vacina e a sua dose dentro do esquema vacinal indicado ${ }^{(14)}$.

Em estudo realizado em Montes Claros-MG, o aprazamento foi identificado em 5(27,7\%) de 18 salas vacinas observadas, sendo constatado que a maioria dos profissionais realizava a aplicação de maneira incorreta $^{(15)}$. Outro estudo realizou observação de 116 CSC e identificou que 114(98,3\%) CSC estavam aprazados $^{(16)}$. Os profissionais, quando comprometidos com as recomendações do PNI, registram o aprazamento de vacinas. Tal fato não foi observado neste, pois não houve o registro do aprazamento na totalidade dos cartões de vacinação observados.

No estudo ${ }^{(13)}$ de Recife-PE, observou-se que a maioria dos profissionais $17(94,4 \%$ ) orientou sobre as vacinas administradas. Em estudo ${ }^{(4)}$ realizado em duas salas de vacinas de dois municípios de pequeno porte do estado de São Paulo, observou-se que nenhum dos 48 usuários receberam orientação do profissional sobre o retorno para completude do esquema vacinal. Nas observações realizadas deste estudo, identificou-se que em $82,1 \%$ dos atendimentos as vacinadoras reforçaram verbalmente o retorno do usuário para completude do esquema vacinal. A orientação do retorno evidencia-se como essencial para adesão dos usuários ao calendário vacinal que confere a proteção e prevenção às doenças imunopreveníveis, bem como para o alcance da meta de cobertura vacinal.

As POVs são consideradas quando um usuário sem contraindicações à vacinação busca o serviço de 
saúde e não recebe as vacinas em consonância com o calendário de esquema vacinal. Dentre os motivos para não vacinação, destacam-se: falsas contraindicações, atitude dos profissionais de enfermagem, atitude dos usuários, indisponibilidade de profissionais capacitados e problemas logísticos dos serviços no provimento de imunobiológicos e insumos ${ }^{(13)}$. Porém, no município do estudo, todas as vacinas e insumos estavam disponíveis no momento das observações, o que não justifica a ocorrência de POVs nas salas de vacina, identificados em 12(11,3\%) ocorrências.

O registro correto da administração de vacinas pelos profissionais de enfermagem ocorreu em 98,1\% e $84 \%$ das observações nesta pesquisa, respectivamente, no cartão do usuário e no SIPNI. O registro deve ser realizado de forma completa e correta no cartão do usuário e no SIPNI de forma a fornecer informações fidedignas e assim retratar a situação de saúde no que tange à imunização da população por doenças imunopreveníveis ${ }^{(10)}$. O registro no cartão do usuário deve conter os seguintes dados: data, lote e laboratório da vacina, e assinatura do profissional. No SIPNI, o registro contempla o nome do usuário pelo cartão SUS, a vacina, lote, laboratório, dose do esquema vacinal e o profissional( ${ }^{(5)}$.

No município do estudo a disponibilidade de imunobiológicos e insumos para as salas de vacina decorrem da gestão da coordenação articulada com as gerências das unidades de saúde e dos profissionais de enfermagem atuantes em salas de vacinas. Esses profissionais conhecem a demanda e se organizam para atender com êxito, observando as necessidades de imunobiológicos e insumos, pois todas as vacinas procuradas pelos usuários estavam disponíveis para aplicação. Tal dado difere do encontrado no estudo em Montes Claros-MG que, das 18 salas de vacinas, em 17(94,4\%) houve a falta de algum imunobiológico ${ }^{(15)}$.

O registro da temperatura do refrigerador nas salas de vacinas foi avaliado no estudo realizado na macrorregião oeste de saúde de Minas Gerais, em que das 253 salas de vacinas, $70,8 \%$ havia o registro adequado do monitoramento da temperatura do refrigerador ${ }^{(17)}$. Outro estudo observacional em 41 salas de vacina no interior de São Paulo identificou que em $90,2 \%$ das salas de vacina havia a leitura e o registro correto da temperatura do refrigerador no início e fim da jornada de trabalho ${ }^{(18)}$. Nas observações neste estudo, verificou-se que esta anotação foi realizada como rotina diária em 75,5\%, o que compromete a precisão da qualidade dos insumos armazenados ofertados à população.

A temperatura em que devem ser armazenados os imunobiológicos deve estar entre $2^{\circ} \mathrm{C}$ a $8^{\circ} \mathrm{C}$, e sua leitura deve ser realizada no horário de início e fim do expediente, sendo registrada no boletim de controle diário de temperatura que, preferencialmente, deve estar fixado externamente na porta do refrigerador ${ }^{(19)}$.

Em estudo realizado em salas de vacinas ${ }^{(19)}$, verificaram-se os mapas de registro de temperatura do refrigerador, os quais indicaram que as temperaturas estavam inadequadas quando observado o padrão de acondicionamento dos imunobiológicos. Nesta pesquisa, em 81,1\% das observações realizadas a temperatura da geladeira estava adequada ao padrão preconizado pelo PNI. Essa inadequação na temperatura do refrigerador ocasiona uma preocupação com a qualidade dos imunobiológicos ofertados aos usuários do serviço ${ }^{(19)}$.

A abertura dos frascos multidoses requer a rotulagem do frasco, sendo isto preconizado pelo PNI. O registro do rótulo de abertura do frasco deve conter a data e hora da abertura do frasco e a fixação na ampola de vacina, desde que não comprometa as informações do rótulo da indústria farmacêutica e da visualização do conteúdo interno ${ }^{(14)}$. Em estudo realizado em salas de vacina da rede pública em Marília-SP, em todas as 41 salas de vacinas observadas os frascos estavam rotulados ${ }^{(11)}$, dado que difere do encontrado neste estudo, em que os frascos estavam rotulados em 74,4\% das observações.

O registro do boletim de movimentação deve ser preenchido na rotina da sala de vacina, uma vez que esses dados serão transmitidos no SIPNI no módulo movimento de imunobiológicos. Nesta pesquisa, os boletins de movimentação estavam preenchidos em apenas $16 \%$ das observações. Essa ação tem o objetivo de subsidiar o planejamento e programação dos imunobiológicos e insumos, considerando a disponibilidade na rede pública e privada, além de auxiliar no controle do estoque(5).

Aliado ao controle de fluxo de insumos no SIPNI, agrega-se o controle do estoque pelo SIES, sendo que neste estudo, em $55,7 \%$ das observações, o estoque estava atualizado conforme os insumos disponíveis na sala de vacina. No entanto, preconiza-se que o estoque deve ser atualizado online, pois isso organiza a logística de distribuição das solicitações realizadas ${ }^{(6)}$. 
Porém, para gerenciar tantas informações e obter um bom resultado, é imprescindível que se tenha um bom sistema de armazenamento, manipulação e distribuição. A necessidade do controle de estoque em uma instituição é fundamental para a qualidade do serviço prestado à população, de forma a promover a excelência do serviço de acordo com o princípio da eficiência ${ }^{(20)}$.

O programa SIPNI está em atualização constante pelo Departamento de Informática do Ministério de Saúde, pois carece de melhorias para atender a diversas demandas, como a introdução de novos imunobiológicos e insumos, e avanços nos fluxos operacionais e a integração com outros sistemas de informação. Essas atualizações são denominadas de pacotes de atualizações do SIPNI para utilização no desktop ${ }^{(5)}$.

As limitações do estudo decorreram do fato de utilizar a observação na coleta de dados sobre as práticas dos profissionais de enfermagem atuantes em salas de vacinas relacionados aos registros, de uma pesquisadora ser a coordenadora do PNI do município e da temporalidade das observações por sala de vacinas.

\section{- CONCLUSÃO}

Por meio das observações, verificou-se a realização dos registros de dados sobre o uso de imunobiológicos e insumos pelos profissionais de enfermagem atuantes em salas de vacinas. Como panorama favorável, destacam-se procedimentos como: registro do usuário no PNI, disponibilidade de informativo do calendário de esquema vacinal, aprazamento e orientação do retorno de vacinas aprazadas, registro da vacina aplicada no cartão de vacina do usuário e SIPNI, disponibilidade das vacinas na rede pública, cuidados no armazenamento de vacinas no refrigerador e dos frascos multidoses.

Por outro lado, vislumbram-se oportunidades de melhorias no processo de trabalho quanto à adesão populacional para aplicação da vacina na faixa etária recomendada, à discussão sobre a necessidade de adiamento na aplicação de vacinas, ao registro de movimentação do imunobiológico no SIPNI e SIES, à manutenção do estoque atualizado no SIES e à utilização da versão atualizada do programa SIPNI. Recomenda-se a realização de ações educativas no serviço da atenção básica do município, de forma a contemplar as lacunas de conhecimento existentes sobre os registros no SIPNI e SIES.

Ademais, sugere-se avaliar a aplicabilidade e validade de instrumentos como os procedimentos operacionais padrão em salas de vacinas para a mobilização, discussão, elaboração e atualização do incremento na prática diária dos profissionais. O uso desses instrumentos possibilita ampliar a segurança dos profissionais no que tange aos registros, de forma a aprimorar e qualificar o exercício laboral da enfermagem.

As limitações do estudo decorreram de utilizar a observação na coleta de dados sobre as práticas dos profissionais de enfermagem atuantes em salas de vacinas relacionados aos registros, de uma pesquisadora ser a coordenadora do PNI do município e da temporalidade das observações por sala de vacinas.

Este estudo contribui para as práticas em sala de vacinas, uma vez que as observações contemplaram os programas utilizados em todo território nacional no âmbito do SUS, sendo observados os registros realizados pelos profissionais, os quais carecem de educação permanente no SIPNI e SIES.

\section{REFERÊNCIAS}

1. Guimarães TMR, Alves JGB, Tavares MMF. Impacto das ações de imunização pelo Programa Saúde da Família na mortalidade infantil por doenças evitáveis em Olinda, Pernambuco, Brasil. Cad. Saúde Publica. [Internet]. 2009 [acesso em 2017 jul 11]; 25(4). Disponível em: http://dx.doi.org/10.1590/S0102-311X2009000400018.

2. Homma A, Martins RM, Leal MLF, Freire MS, Couto AR. Atualização em vacinas, imunizações e inovação tecnológica. Cienc. saúde coletiva. [Internet]. 2011 [acesso em 2017 jul 11]; 16(2). Disponível em: http://dx.doi. org/10.1590/S1413-81232011000200008. 
3. Tavares RE, Tocantins FR. Nursing actions in primary care and the control of diseases preventable through vaccines. Rev. Bras. Enferm. [Internet]. 2015 [acesso em 2018 abr 14]; 68(5). Disponível em: http://dx.doi. org/10.1590/0034-7167.2015680506i.

4. Fossa AM, Protti AM, Rocha MCP, Horibe TM, Pedroso GER. Conservação e administração de vacinas: a atuação da enfermagem. Saude Rev. [Internet]. 2015 [acesso em 2017 ago 04]; 15(40). Disponível em: http://dx.doi. org/10.15600/2238-1244/sr.v15n40p85-96.

5. Fundação de Vigilância em Saúde do Amazonas (FVS-AM). Sistema de Insumos Estratégicos-GIES. [Internet]. Amazonas: FVS-AM; 2012 [acesso em 2016 abr 21]. Disponível em: http://www.fvs.am.gov.br/images/pdf/giescartilha-sistema-insumos.pdf.

6. Ministério da Saúde (BR). Coordenação Geral do Programa Nacional de Imunizações. Manual do Sistema de Informação do Programa Nacional de Imunizações SIPNI. [Internet] Brasília: Ministério da Saúde; 2014 [acesso 2017 ago 04]. Disponível em: http://www.saude.campinas.sp.gov.br/vigilancia/vacinacao/2016/manual_SIPNI_ fev_2014.pdf.

7. Hulley SB, Cummings SR, Browner WS, Grady DG, Newman TB. Delineando a pesquisa clínica: uma abordagem epidemiológica. 4. ed. Porto Alegre: Artmed; 2015.

8. Marconi MA, Lakatos EM. Fundamentos de Metodologia Científica. 7. ed. São Paulo: Atlas; 2010.

9. Ministério da Saúde (BR). Conselho Nacional de Saúde. Diretrizes e normas regulamentadoras de pesquisa envolvendo seres humanos. Resolução n. 466, de 12 de dezembro de 2012. Brasília; 2012.

10. Barasuol MT. Análise da cobertura vacinal do sistema de informação do programa de imunizações na secretaria de saúde de Augusto Pestana [monografia]. Porto Alegre: Universidade Federal do Rio Grande do Sul; 2015.

11. Vasconcelos CEV, Rocha SA, Ayres JA. Avaliação normativa das salas de vacinas na rede pública de saúde do Município de Marília, Estado de São Paulo, Brasil, 2008-2009. Epidemiol. Serv. Saúde. [Internet]. 2012 [acesso em 2017 jul 14]; 21(1). Disponível em: http://dx.doi.org/10.5123/S1679-49742012000100017.

12. Casarin ST, Oliveira ESA, Ceolin T, Soares SD. Cuidados prestados pela equipe de enfermagem no atendimento aos eventos adversos pós-vacinais. Rev. Enferm UFPI. [Internet]. 2016 [acesso em 2017 jul 20]; 5(2). Disponível em: https://doi.org/10.26694/reufpi.v5i2.527.

13. Barros MGM, Santos MCS, Bertolini RPT, Andrade M. Perda de oportunidade de vacinação: aspectos relacionados à atuação da atenção primária em Recife, Pernambuco, 2012. Epidemiol. Serv. Saúde. [Internet]. 2015 [acesso em 2017 ago 04]; 24(4). Disponível em: http://dx.doi.org/10.5123/S1679-49742015000400012.

14. Ministério da Saúde (BR). Secretaria de Vigilância em Saúde. Departamento de Vigilância das Doenças Transmissíveis. Manual de Normas e Procedimentos para Vacinação. [Internet]. Brasília: Ministério da Saúde; 2014 [acesso em 2017 ago 08]. Disponível em: http://bvsms.saude.gov.br/bvs/publicacoes/manual_procedimentos_ vacinacao.pdf.

15. Siqueira LG, Martins AMEBL, Versiani CMC, Almeida LAV, Oliveira CS, Nascimento JE, et al. Avaliação da organização e funcionamento das salas de vacina na atenção primária à saúde em Montes Claros, Minas Gerais, 2015. Epidemiol. Serv. Saúde [Internet]. 2017 [acesso em 2018 abr 15]; 26(3). Disponível em: https://doi.org/10.5123/ S1679-49742017000300013. 
16. Vieira DS, Santos NCCB, da Costa DKG, Pereira MM, Vaz EMC, Reichert APS. Registro de ações para prevenção de morbidade infantil na caderneta de saúde da criança. Ciênc. saúde coletiva. [Internet]. 2016 [acesso em 2018 abr 18]; 21(7). Disponível em: https://doi.org/10.1590/1413-81232015217.09442015.

17. Oliveira VC, Gallardo MDPS, Arcêncio RA, Gontijo TL, Pinto IC. Avaliação da qualidade de conservação de vacinas na atenção Primária à saúde. Ciênc. saúde coletiva. [Internet]. 2014 [acesso em 2017 jul 02]; 19(9). Disponível em: http://dx.doi.org/10.1590/1413-81232014199.12252013.

18. Vasconcelos CEV, Rocha SA, Ayres JA. Avaliação normativa das salas de vacinas na rede pública de saúde do Município de Marília, Estado de São Paulo, Brasil, 2008-2009. Epidemiol. Serv. Saúde. [Internet]. 2012 [acesso em 2017 jul 14]; 21(1). Disponível em: http://dx.doi.org/10.5123/S1679-49742012000100017.

19. Queiroz AS, Moura ERF, Nogueira PSF, Oliveira NC, Pereira MMQ. Atuação da equipe de enfermagem na sala de vacinação e suas condições de funcionamento. Rev. Rene. [Internet]. 2009 [acesso em 2017 jul 03]; 10(4). Disponível em: www.revistarene.ufc.br/vol10n4_pdf/v10n4a15.pdf.

20. Rocha GR, Gonçalves SDM, Silva PLN, Veloso MAA. Avaliação da aplicabilidade de ferramentas de gestão em uma Unidade Básica de Saúde. Revgestsist saúde. [Internet]. 2015 [acesso em 2018 abr 18]; 4(2). Disponível em: http://dx.doi.org/10.5585/rgss.v4i2.163. 